Original Research

\title{
Epidemiology, clinical characteristics and severity of gradual onset injuries in recreational road cyclists: A cross-sectional study in 21,824 cyclists - SAFER XIII
}

\author{
François du Toit a, Martin Schwellnus b, c, *, Paola Wood ${ }^{\text {a }}$, Sonja Swanevelder ${ }^{\text {, }}$, \\ Jannelene Killops ${ }^{\text {b }}$, Esme Jordaan ${ }^{\mathrm{d}, \mathrm{e}}$ \\ ${ }^{a}$ Sport, Exercise Medicine and Lifestyle Institute (SEMLI) and Division of Biokinetics and Sports Science, Department of Physiology, Faculty of Health \\ Sciences, University of Pretoria, South Africa \\ b Sport, Exercise Medicine and Lifestyle Institute (SEMLI), Faculty of Health Sciences, University of Pretoria, South Africa \\ ${ }^{c}$ International Olympic Committee (IOC) Research Centre, South Africa \\ d Biostatistics Unit, South African Medical Research Council, South Africa \\ e Statistics and Population Studies Department, University of the Western Cape, South Africa
}

\section{A R T I C L E I N F O}

\section{Article history:}

Received 11 March 2020

Received in revised form

6 August 2020

Accepted 10 August 2020

\section{Keywords:}

Gradual onset injuries

Overuse injuries

Epidemiology

Severity of injuries

\begin{abstract}
A B S T R A C T
Objectives: Prevalence, clinical characteristics and severity of gradual onset injuries (GOIs) in cyclists are poorly documented. We determine the prevalence, anatomical regions/sites affected and severity of GOIs among entrants in a community-based mass participation event.

Design: Cross-sectional study;

Setting: Cape Town Cycle Tour;

Participants: Race entrants.

Main outcome measures: Of 35,914 entrants, 27,349 completed pre-race medical questionnaires. We studied 21,824 consenting cyclists (60.8\% of entrants). Crude lifetime prevalence, retrospective annual incidence, anatomical region/sites, specific GOI, tissue type and GOI severity is reported.

Results: The lifetime prevalence of GOIs was $2.8 \%$, with an annual incidence of $2.5 \%$. More common anatomical regions affected by GOIs were lower limb (43.4\%), upper limb (19.8\%), and lower back (11.5\%). The knee (26.3\%), shoulder (13\%), and lower back (11.5\%) regions were mostly affected. The most common GOI was anterior knee pain (14.2\%). Of the GOIs, 55\% were in soft tissue. 50\% of cyclists reported symptom duration $>12$ months, and $37.3 \%$ of GOIs were severe enough to reduce/prevent cycling. Conclusion: $2.5 \%$ recreational cyclists report a GOI annually. $>50 \%$ of GOIs affect the knee, lower back and shoulder. GOIs negatively affect cycling. Risk factors related to GOIs in cyclists need to be determined to develop and implement prevention programs.
\end{abstract}

(C) 2020 Elsevier Ltd. All rights reserved.

\section{Introduction}

The burden of non-communicable diseases (NCDs) of lifestyle can be reduced by a healthy lifestyle that includes participation in regular moderate-to high-intensity physical activity for $>150 \mathrm{~min} /$ week (Bahr et al., 2020; Khan et al., 2012; Kohl et al., 2012). Mass community-based participation in sport and endurance events,

\footnotetext{
* Corresponding author. Sport, Exercise Medicine and Lifestyle Institute (SEMLI), Faculty of Health Sciences, University of Pretoria, Sports Campus, Burnett Street, Hatfield, Pretoria, 0020, South Africa.

E-mail address: martin.schwellnus@up.ac.za (M. Schwellnus).
}

such as cycling, is therefore encouraged as part of a healthy lifestyle and a substantial growth in race entrants has been observed in South African events such as the annual $109 \mathrm{~km}$ Cape Town Cycle Tour (CTCT) and multi-stage mountain-bike races (Cape Epic, Wines to Whales).

Cycling injuries can be acute/traumatic injuries caused by falls or injuries can be of a more gradual onset. As cycling gains popularity there may be an increased number of injuries amongst both amateur and professional cyclists (Clarsen et al., 2010; Dettori \& Norvell, 2006; Kotler et al., 2016). The prevalence of gradual onset cycling injuries is as high as 85\% (Dettori \& Norvell, 2006). Other SAFER studies have focused on the traumatic injuries in 
cyclists (Killops et al., 2020). Gradual onset injuries (GOIs) in cyclists are associated with long, repetitive training sessions (Clarsen et al., 2013; Decalzi et al., 2013), thus this paper will focus on the GOIs in cyclists.

The epidemiology of GOIs in cyclists is not well described. In one review, dating back to 2006, it was reported that GOIs in cyclists mainly affect the knee, ankle, back, neck/shoulders, hands/wrists, and the buttocks/perineum (Dettori \& Norvell, 2006). More recently, the epidemiology of GOIs among professional cyclists that attend training camps was studied (Clarsen et al., 2010). In this study, lower back pain, anterior knee pain and neck-shoulder pain were found to be the most frequently reported GOIs. Recreational or amateur cyclists make up the largest number of cyclists, yet there are few studies reporting the epidemiology GOIs in this population. In one study, buttock pain, knee injuries, specifically 'patellar pain syndrome' and neck-shoulder pain were common among amateur cyclists during a $\geq 5$ days cycle tour (Clarsen et al., 2010; Weiss, 1985), In a more recent study, $88 \%$ of amateur cyclist participating in a 1-day $94.7 \mathrm{~km}$ cycle challenge reported a GOI, with the neck, back, hand/worst and buttock/perineum being the common anatomical areas affected (Van der Walt et al., 2014). However, it is difficult to compare data on the clinical characteristics, including severity, of GOIs in cyclists between studies because of methodological differences in recording of GOIs (Clarsen et al., 2015) and differences in cycling populations studied e.g. professional and recreational cyclist differ significantly in annual cycling exposure, training history and equipment selection. Other epidemiological studies on GOIs in road cyclists mainly refer to leisure or transportation activities. Currently, little is known about GOIs in massparticipation cycling events that attract recreational cyclists, despite the fact that GOIs are very common (Weiss, 1985; Wilber et al., 1995).

Therefore, the aim of this study was to describe the epidemiology (lifetime prevalence, retrospective annual incidence), clinical characteristics (main anatomical region and specific anatomical sites affected, prevalence of specific common GOIs, and to describe these by tissue type affected), and severity (duration of symptoms and severity grade) in recreational cyclists that entered for a large community-based cycling event.

\section{Methods}

This descriptive cross-sectional study formed part of a series of on-going SAFER (Strategies to reduce Adverse medical events For the Exercise $\underline{\text { R }) ~ s t u d i e s ~(S c h w e l l n u s ~ \& ~ D e r m a n, ~ 2014) . ~ T h e ~ R e s e a r c h ~}$ Ethics Committee of the Faculty of Health Sciences approved the study (REC numbers 431/2015 and 213/2017). Data collection for the SAFER studies is ongoing, and this study was an analysis of data collected over a one-year period (2016).

A total of 35,914 cyclists entered the $109 \mathrm{~km}$ Cape Town Cycle Tour (CTCT) in 2016, and 21,902 cyclists gave informed consent for their data to be used for research purposes. In 78 cyclists there was missing or incomplete data, therefore, the final study population was 21,824 ( $60.8 \%$ of all cycle race entrants).

All cycle race entrants from the 2016 CTCT were required to complete an online medical questionnaire at the time of registration. The online medical screening tool consisted of a series of questions that were developed to specifically provide clinical information for medical staff in order to improve medical care on race day. The main sections of the medical screening tool were based on the guidelines for cardiovascular evaluation of middle-aged/senior individuals engaging in leisure-time sport activities (Position stand from the European Association of Cardiovascular Prevention and Rehabilitation) (Borjesson et al., 2011), and adapted from our previous studies in distance runners (Rotunno et al., 2018; Schwabe et al., 2018). The detailed methodology of the online medical screening tool development and implementation was described in previous studies amongst endurance runners (Rotunno et al., 2018; Schwabe et al., 2018). Additional questions, specifically related to common medical complications encountered during cycling, were added. The final screening questions related to the cyclists' training and medical history including history of acute traumatic and GOIs (current, or in the last 12 months).

In keeping with a recent IOC consensus paper (Bahr et al., 2020), we recognise that cycling injuries result from a transfer of kinetic energy (agent) that damages tissue. This transfer of energy may result from: 1) a near-instantaneous exchange of large quantities of kinetic energy (e.g. cycling accident), 2) from the gradual accumulation of low-energy transfer over time (as in the development of patellofemoral pain), or 3) from a combination of both mechanisms (repetitive training regime resulting in discal lower back pain that then manifests itself acutely following a minor cycling accident) (Bahr et al., 2020). We agree that mode of onset for injuries should be conceptualized as a continuum interplay of energy exposures. However, in our medical screening tool, which was designed prior to the recent IOC consensus statement (Bahr et al., 2020), cyclists were specifically asked the following question related to injuries: "Do you or did you suffer from any symptoms of a CHRONIC (no accident) cycling injury (muscles tendons bones ligaments or joints) IN YOUR CYCLING CAREER?“. We therefore included only injuries where there was no self -reported history of a nearinstantaneous exchange of large quantities of kinetic energy (e.g. cycling accident), and we report these as GOIs. The severity of a GOI was defined as: "An injury that is/was severe enough to interfere with cycling or require treatment e.g. use medication or require you to seek medical advice from a health professional".

In response to a "yes" answer to this question, participants were required to complete additional questions with drop-down boxes that were related to the GOIs, including: past or current injury, side of the body the injury occurred (if applicable), anatomical site, type of anatomical structure, severity, duration of symptoms, and whether the injury was one of the more commonly known GOIs. A GOI was reported by 617 cyclists and 29 cyclists reported more than one GOI (total of 646 GOIs).

In this study, we report the following epidemiological outcome variables in the 617 cyclists reporting a GOI: lifetime prevalence of GOIs (\% cyclists reporting a GOI in their lifetime career of cycling), and retrospective annual incidence of GOICs (\% cyclists reporting a GOI in the past 12 months). The lifetime prevalence of GOIs was determined in response to a yes answer for "an injury in the cyclists cycling career". The retrospective annual incidence of GOIs was determined in response to a yes answer for "an injury in the past 12 months or currently".

The clinical characteristic outcome variables for 646 GOIs were as follows: main anatomical region and specific anatomical site of GOIs, specific common GOIs and GOIs by tissue type affected (muscle, tendon, ligament, bone, joint, nerve).

We report on the severity of GOIs $(n=646)$ as follows: duration of symptoms (0-3 months, 4-12 months and $>12$ months), and an injury severity grading (Grade I-IV) commonly used for "overuse" injuries in sport (Taunton et al., 2003). For the purposes of comparing the grading of injuries to that of other studies, we also report GOIs severity in two categories of severity: a) less severe, where cyclists are still able to cycle and compete with no or minimal interference (Grade I and Grade II), and b) more severe, where the GOIs interferes with the cyclists' ability to, and even stop them from, training or competing (Grade III and Grade IV).

All questionnaire data were entered into an Excel spreadsheet (Microsoft 2010) and analysed using the SAS v9.4 statistical program. Only data for consented cycle race entries were used for 
analysis ( $\mathrm{n}=21824)$. The lifetime prevalence of GOIs (muscles, tendons, bones, ligaments or joints), the retrospective annual incidence of GOIs and the point prevalence are reported, considering the correlation resulting from the same cyclist reporting multiple injuries.

GOIs in the main anatomical region, specific anatomical sites, specific common GOIs, tissue and type of GOIs are described with frequencies and percentages out of all GOIs. The duration of symptoms (months) of GOIs are categorized as of 0-3 months, 4-12 months, and >12 months, and the frequency (\%) is reported for each category overall, as well as by main anatomical region.

\section{Results}

Although the response rate was acceptable, a post-hoc analysis was conducted to determine if the participants in this study were indeed representative of all the cycle race entrants. The demographics (age and gender) of all cyclists entering the 2016 CTCT are compared with the consenting cycle race entrants that were participants in this study (Table 1 ).

We note that, in our study population, there were significantly more cyclists over 50 years old ( $<<0.0001$ ), as well as a higher \% of male cyclists $(\mathrm{p}=0.0390)$, compared with all cycle race entrants (Table 1).

The point prevalence (at the time of race registration) of GOI in this population $(\mathrm{n}=21824$ ) was $1.1 \%$ (95\%CI: $0.9-1.2 ; n=230)$, the lifetime prevalence of GOIs was 2.8\% (95\%CI: $2.6-3.1 ; \mathrm{n}=617)$, and the retrospective annual incidence of GOIs was $2.5 \%(95 \% \mathrm{CI}$ : $2.3-2.7 ; \mathrm{n}=551$ ).

The frequency (\%) of GOIs by main anatomical region and specific anatomical sites is depicted in Table 2.

The main anatomical region of GOIs (as a \% of all GOIs) was the lower limb region (43.4\%), followed by the upper limb (19.8\%), lower back (11.5\%), and hip/groin/pelvis (10.7\%). Within the main anatomical regions, the three most affected specific anatomical sites for GOIs were the knee (26.3\%), shoulder (13\%), and the lower back (11.5\%).

A list of common reported GOIs were included in the questionnaire and for $76.3 \%(n=609)$ of injuries, cyclists reported a specific common GOI. The frequency (\%) of specific common GOIs is listed in Table 3.

The four most specific common GOIs asked were anterior knee pain (AKP)/Patellofemoral pain (PFP) (14.2\%), lower back pain (LBP) (10.5\%), shoulder injury (9.4\%) and the iliotibial band friction syndrome (ITBFS) (8.5\%) respectively.

The frequency (\%) of GOIs by tissue type is depicted in Supplementary Table S1. In $98.8 \%$ of injuries $(n=637)$ the cyclist reported a tissue type. The majority of GOIs were to the soft tissues (muscle, tendon, ligament and nerve) (55\%), and 35.6\% of GOIs were reported as musculotendinous. The specific tissue type in which cyclists reported GOIs (\% of all injuries) were muscle (19\%), followed by bone (18.9\%), and tendon (16.6\%).
Table 2

Gradual onset injuries (GOIs) in cyclists by anatomical region and specific anatomical sites of GOIs (expressed as \% of all GOIs) $(n=646)$.

\begin{tabular}{|c|c|c|c|}
\hline Main anatomical region & Specific anatomical sites & $\mathrm{n}$ & $\%$ of all GOIs \\
\hline \multirow[t]{4}{*}{ Head, Neck \& Face } & All & 37 & 5.7 \\
\hline & Neck & 28 & 4.3 \\
\hline & Head & 7 & 1.1 \\
\hline & Face & 2 & 0.3 \\
\hline \multirow[t]{7}{*}{ Upper Limbs } & All & 128 & 19.8 \\
\hline & Shoulder & 84 & 13.0 \\
\hline & Wrist & 16 & 2.5 \\
\hline & Elbow & 15 & 2.3 \\
\hline & Finger & 11 & 1.7 \\
\hline & Upper arm & 1 & 0.2 \\
\hline & Forearm & 1 & 0.2 \\
\hline \multirow[t]{3}{*}{ Trunk/Chest } & All & 16 & 2.5 \\
\hline & Back chest & 9 & 1.4 \\
\hline & Front chest & 7 & 1.1 \\
\hline Lower Back & All & 74 & 11.5 \\
\hline \multirow[t]{4}{*}{ Hip/Groin/Pelvis } & All & 69 & 10.7 \\
\hline & Hip & 32 & 5.0 \\
\hline & Hip muscle \# & 26 & 4.0 \\
\hline & Groin & 11 & 1.7 \\
\hline \multirow[t]{9}{*}{ Lower Limbs } & All & 281 & 43.4 \\
\hline & Knee & 170 & 26.3 \\
\hline & Achilles & 22 & 3.4 \\
\hline & Hamstring muscle & 18 & 2.8 \\
\hline & Foot & 18 & 2.8 \\
\hline & Calf muscle & 17 & 2.6 \\
\hline & Ankle & 17 & 2.6 \\
\hline & Quadriceps muscle & 11 & 1.7 \\
\hline & Shin/Lower leg & 8 & 1.2 \\
\hline Other & All & 41 & 6.4 \\
\hline
\end{tabular}

$\mathrm{n}$ : number of injuries reported in the study.

\% Gradual onset injuries frequency (\%) of reported injuries in the study. \# including gluteal (buttock) muscles.

A further analysis of the frequency (\%) of GOIs by type of tissue in the main anatomical region is depicted in Supplementary Table S2. The most common tissue affected by GOIs in the lower limb was tendon (26.3\%), whilst bone was the most common tissue affected by GOIs in the upper limb (32\%) and trunk/chest (75\%).

Cyclists also reported the duration of symptoms of the GOI, and this was reported in $76.3 \%(n=609)$ of injuries. The duration of symptoms was categorized as follows: 0-3 months, 4-12 months, and $>12$ months (Supplementary Figure S1). In $47.8 \%$ of GOIs, symptoms lasted $>12$ months. This was followed by a symptom duration of $4-12$ months in $33.8 \%$ of GOIs, and only in $18.4 \%$ of the GOIs were symptoms present for $<3$ months.

The frequency (\%) of reported GOIs in each main anatomical region by duration of symptoms category is depicted in Supplementary Table S3. Of the reported GOIs by main anatomical region, 63.8\% hip/groin/pelvis, 59.5\% head/neck/face, and 59.5\% lower back injuries were reported as having a symptom duration of $>12$ months. The symptom duration was shorter for some anatomical regions than for others $(\mathrm{p}=0.003)$. On average, the duration of symptoms of GOIs was shorter in the trunk/chest region

Table 1

Characteristics of all cycle race entrants and study participants (consenting cycle race entrants).

\begin{tabular}{|c|c|c|c|c|c|c|}
\hline \multirow[t]{2}{*}{ Characteristics } & \multirow{2}{*}{$\frac{\text { All cycle race entrants }(\mathrm{n}=35914)}{\mathrm{n}}$} & \multirow{2}{*}{$\frac{\text { Entrants consenting as study participants }(\mathrm{n}=21824)}{\%}$} & \multicolumn{2}{|l|}{$\mathrm{p}$} & & \\
\hline & & & $\mathrm{n}$ & $\%$ & & \\
\hline \multirow[t]{2}{*}{ Gender } & Males & 28311 & 78.8 & 17282 & 79.2 & $\mathrm{p}=0.0390$ \\
\hline & Females & 7603 & 21.2 & 4542 & 20.8 & \\
\hline \multirow[t]{4}{*}{ Age groups (years) } & $\leq 30$ & 6453 & 18 & 3333 & 15.3 & $\mathrm{p}<0.0001$ \\
\hline & 31 to $\leq 40$ & 7814 & 21.8 & 4447 & 20.4 & \\
\hline & 41 to $\leq 50$ & 10583 & 29.4 & 6382 & 29.2 & \\
\hline & $>50$ & 11064 & 30.8 & 7662 & 35.1 & \\
\hline
\end{tabular}

p: p-value - all cycle race entrants vs. entrants consenting as study participants. 
Table 3

Specific common gradual onset injuries (GOIs) (expressed as \% of all GOIs) ( $\mathrm{n}=646$ ).

\begin{tabular}{lll}
\hline Specific common gradual onset injuries (GOIs) in cyclists & $\mathrm{n}$ & \% (of all GOIs) \\
\hline Knee - Anterior knee pain (AKP)/Patellofemoral pain (PFP) & 92 & 14.2 \\
Lower back pain (LBP) & 68 & 10.5 \\
Shoulder pain & 55 & 9.4 \\
Knee - Iliotibial band friction syndrome (ITBFS) & 30 & 8.5 \\
Hip joint pain & 28 & 4.7 \\
Neck pain & 23 & 4.3 \\
Hip muscle injury (including gluteus/buttock muscles) & 22 & 3.6 \\
Achilles tendon injury & 21 & 3.4 \\
Numbness in the hand/fingers & 19 \\
Foot or heel pain & 16 \\
Calf muscle injury & 16 \\
Hamstring injury & 15 \\
Elbow pain & 10 \\
Wrist pain & 5.3 \\
Quadriceps muscle injury & 2.9 \\
Groin/genital numbness & 2.5 \\
Saddle sores & 2.5 \\
Other & 6 \\
Injuries where specific commonly reported GOIs data were missing & 6 \\
\hline
\end{tabular}

$\mathrm{n}$ : number of injuries reported in the study.

$\%$ Gradual onset injuries frequency (\%) of reported injuries in the study.

$(\mathrm{p}=0.0002)$ and longer for hip/groin/pelvis $(\mathrm{p}=0.006)$ and lower back ( $p=0.039$ ) regions compared to the other anatomical regions.

The frequency (\%) of GOIs severity (grade I-IV) is depicted in Supplementary Table S4. Of the reported injuries, 93.2\% $(n=602)$ reported severity grading and out of those, $>37 \%$ were severe enough to interfere with the cyclist's ability to, and even stop them from, training or competing.

The frequency (\%) of GOIs severity (grade I-IV) by main anatomical region is depicted in Supplementary Table S5 (\% of reported GOIs by main anatomical region). The severity grading was similar for all anatomical regions, with no one region reporting a more severe grading compared to the other regions $(\mathrm{p}=0.616)$.

\section{Discussion}

The main findings of this study were 1) $2.8 \%$ of recreational cyclists reported a GOI in their cycling career (about 1 in 35 cyclists), with an annual incidence of $2.5 \%$, and a $1.1 \%$ point prevalence at the time of race registration 2) the lower limb was the most commonly affected by GOIs (43.4\%), followed by the upper limb (19.8\%), lower back (11.5\%), and hip/groin/pelvis (10.7\%), 3) the three most affected specific anatomical sites of GOIs were the knee (26.3\%), shoulder (13\%), and the lower back (11.5\%), and the most commonly reported specific GOI was AKP/PFP (14.2\%), 4) 55\% of GOIs were soft tissue injuries (muscle, tendon, ligament and nerve), and 5) almost $50 \%$ of cyclists with a GOI reported a symptom duration of $>12$ months, 6 ) almost $60 \%$ of hip/groin/pelvis, head, neck and face, and lower back GOIs were reported as having a symptom duration of $>12$ months, and 7) $>37 \%$ of GOIs were severe enough to reduce or prevent cycling (Grades III and IV).

Although the epidemiology of GOIs has been studied (Barrios et al., 2015; Clarsen et al., 2010; Dahlquist et al., 2015), there are substantial methodological differences between studies making comparisons between studies difficult. Methodological differences include: differences in terminology and the definition of gradual onset injuries (Dahlquist et al., 2015; Van der Walt et al., 2014; Wilber et al., 1995), defining and reporting the exposure (annual incidence, incidence during single-day or multi-day staged races), small sample sizes (Dahlquist et al., 2015; Wilber et al., 1995), lowresponse rates with possible selection bias (Van der Walt et al., 2014; Wilber et al., 1995), differences in the populations studied (ranging from professional cyclists to multistage cycling events)
(Barrios et al., 1997, 2015; Clarsen et al., 2010; De Bernardo et al., 2012), and use of self-reported data with differences in timing and content of questionnaire administration (Dahlquist et al., 2015; Van der Walt et al., 2014; Wilber et al., 1995).

The reported annual incidence of GOIs varies between 17 and 88\% (De Bernardo et al., 2012; Van der Walt et al., 2014). However, in most studies, only elite cyclists were studied where the reported annual incidence of GOIs is between 17 and 58\% (Barrios et al., 1997, 2015; Clarsen et al., 2010; De Bernardo et al., 2012). In two studies in recreational cyclists, an annual incidence of $85-88 \%{ }^{13} 11$ has been reported, and we note that this is substantially higher than the annual incidence we report in our population (2.5\%). We attribute this large variation in annual incidence to the following factors: the low response rates with potential selection bias in many studies and variations in the definitions of a GOI used in our study compared to definitions used in other studies. More specifically, in our study, we predefined GOI as injuries "severe enough to interfere with cycling or require treatment e.g. use medication or require you to seek medical advice from a health professional". In the other two studies in recreational cyclists, the definition of GOI included less "severe" injuries and the response rates were low (Van der Walt et al., 2014; Wilber et al., 1995) resulting in possible selection bias and over-reporting of injuries. We are not aware of any studies that report the lifetime prevalence of GOIs in recreational cyclists, and therefore we cannot compare our results to other studies. As a result of these methodological differences, we suggest that a consensus is developed to standardise definitions and methods of reporting GOIs in cyclists, based on the IOC consensus guidelines on methods for recording and reporting of epidemiological data on injury and illness in sports (Bahr et al., 2020).

In our study, the main anatomical regions of GOIs were reported in the lower limb $(67.9 \%-82.7 \%)$, upper limb $(3.8 \%-10.6 \%)$, and lower back (13.4\%-15.1\%) (Barrios et al., 1997, 2015; De Bernardo et al., 2012). We also show that the lower limb is most commonly affected by GOIs, but we report more GOIs in the upper limb and fewer GOIs in the lower back compared with other studies. The precise reasons for these differences are not clear, but could be related to the level and experience of cyclists in our study (recreational) compared with elite cyclists in other studies (Barrios et al., 1997, 2015; De Bernardo et al., 2012).

In our study, the three most affected specific anatomical sites for GOIs were the knee, shoulder, and the lower back and our results are 
very similar to other studies in recreational cyclists: knee $(22 \%-$ $53.8 \%)$, shoulder (17\%-30\%), and lower back (2.7\%-41\%) (Dannenberg et al., 1996; Townes et al., 2005; Van der Walt et al., 2014; Weiss, 1985; Wilber et al., 1995). Other reports also indicate that GOIs affecting the knee are very common and are the most likely cause of time lost due to injury in cyclists (Bini et al., 2014; Kotler et al., 2016; Wanich et al., 2007). In our study, the two most common specific GOIs affecting the knee were AKP/PFP and the ITBFS, and this is similar to other studies (Claes et al., 2015; Deakon, 2012; Farrell et al., 2003). This is a descriptive study and therefore we did not investigate possible mechanisms or causes of these knee injuries. We do recognise that there are multiple possible causal factors for GOIs affecting the knee in cyclists, including repetitive force application, lower limb biomechanical factors such as anatomical malalignment or maltracking, muscle imbalances, and other factors that could affect the response of tissue to repetitive mechanical loading and the rate of healing of microscopic tissue damage. (Lankhorst et al., 2013; Powers et al., 2017). Causal factors need to be explored in future studies.

The frequency of GOIs by tissue type has only been reported in a few studies (Barrios et al., 1997, 2015; De Bernardo et al., 2012). We show that most GOIs were soft tissue injuries and this is consistent with other studies (ranging from $43.4 \%$ to $86 \%$ of GOIs) (Barrios et al., 1997, 2015; De Bernardo et al., 2012). Cycling, by nature, is not associated with high vertical impact forces and changes in acceleration that characterise other sports e.g. running. Therefore, gradual onset bony injuries are less common in cycling and most injuries are in the soft tissues (mostly muscle, tendon and ligament) where force generation and force transfer take place. Gradual onset nerve injuries can be as a result of sustained compression (e.g. sitting on the saddle) or traction (e.g. in the wrist). A direct comparison between frequencies of GOIs and types of GOIs between these studies is again limited by methodological differences.

The severity of GOIs in recreational cyclists has not been wellstudied and there are substantial differences in the classification and definitions of measures of severity of GOIs. Measures of severity grading of GOIs were investigated in six studies (Barrios et al., 1997, 2015; Clarsen et al., 2010; De Bernardo et al., 2012; Van der Walt et al., 2014; Wilber et al., 1995), of which only two were in recreational cyclists (Van der Walt et al., 2014; Wilber et al., 1995). More severe GOIs ranged from $5.7 \%$ to $58.8 \%$ in elite cyclists (Barrios et al., 1997, 2015; Clarsen et al., 2010; De Bernardo et al., 2012), and $16.5 \%-25.5 \%$ in recreational cyclists (Van der Walt et al., 2014; Wilber et al., 1995). In our study, the frequency of more severe GOIs is higher than previous reports in recreational cyclists, but similar to elite cyclists. We also show that the main anatomical region where more severe GOIs were reported are the lower limb, head, neck and face, and the lower back and this is similar to the findings in some (Weiss, 1985), but not all studies (Van der Walt et al., 2014; Wilber et al., 1995). Variation in the definition of "more severe" injuries makes comparison between study results difficult.

A novel finding in our study is that almost $50 \%$ of cyclists with GOIs reported a symptom duration $>12$ months. We are aware of only two studies in recreational cyclists where symptom duration has been reported. In one study, the duration of neurological symptoms (symptoms disappearing immediately after cycling to symptoms for $>1$ week) in the specific anatomical sites was reported (Van der Walt et al., 2014). In another study, the mean duration of symptoms in recreational cyclists who sought medical treatment for GOIs, was 3.7 months (Wilber et al., 1995). We also show that the main anatomical regions where symptom duration $>12$ months is common are hip/groin/pelvis, head, neck and face, and the lower back. We are not aware of any studies in recreational cyclists where symptom duration by main anatomical region has been reported. It is of concern that almost $50 \%$ of cyclists with GOIs report a symptom duration of $>12$ months. The precise reasons for the prolonged symptom duration are not clear and was not explored in this descriptive study. One possible reason is that our definition of a GOI included more severe injuries (as previously mentioned). However, it is also likely that there are multiple other factors at play including that cyclists did not seek attention in cases of mild symptoms that fluctuate in severity, an incorrect diagnosis was made or that treatment was not effective resulting in recurrence of symptoms. Also, in cases of chronic pain, we recognise that nociplastic/algopathic/nocipathic (centralisation of pain) pain may develop after injury and this may also contribute to chronic symptoms in gradual onset injuries (Hainline et al., 2017). A further contributing factor may be that $>64 \%$ of participants were $>40$ years old. We recommend that further research is needed to determine the precise reasons for the prolonged duration of symptoms.

One of the main strengths of this descriptive cross-sectional study is our large sample size. As far as we are aware, it is the largest study describing the epidemiology and clinical characteristics of GOIs in recreational cyclists. Our overall response rate (as a $\%$ of all cycle race entrants) was over $60 \%$, which is high compared with response rates in other similar studies (Van der Walt et al., 2014; Wilber et al., 1995). We were also able to show that our study population was generalizable to all cycle race entrants, but we note that our study population was significantly older, and that male cyclists were significantly more represented in this group. Our results have to be interpreted with this limitation in mind.

One of the most important limitations in our study design is that our data are self-reported. Therefore, there is not only a potential for recall bias, but also that the diagnoses of injuries, including the specific tissue type and the pathology involved could not be verified by clinical assessment or special investigations. This limits the value of these results to describe the epidemiology and clinical characteristics of specific injuries in anatomical areas. However, self-reported data collection in large descriptive epidemiological studies on injuries in athletes is an acceptable and common method (Van der Walt et al., 2014; Weiss, 1985; Wilber et al., 1995), also in numerous studies on cycling injuries (Clarsen et al., 2010, 2014), because athletes seek medical attention for injuries and are generally well-informed about the diagnosis of their injury. Further limitations of our study are that the definition of injury favoured reporting of more severe injuries, the maximum reported duration allowed for injuries was truncated at "> 12 months", which was reported by almost half of the injured cyclists, severity of injuries was not reported for $6.8 \%$ of the reported injuries, and missing data for severity was more prevalent among bone injuries compared to all other tissue type injuries ( $14 \%$ vs $5 \%, \mathrm{p}=0.0010)$. We also acknowledge that data on injury severity are reliant on individual perceptions of pain to determine the grade of severity. Finally, we suggest that a prospective design, currently the gold standard for injury surveillance research, should be used for future studies.

\section{Conclusion}

GOIs in recreational cyclists have not been well studied. We report novel data on the epidemiology, clinical characteristics and severity of GOIs in a large sample of recreational cyclists preparing for a one-day cycling event. About 1 in 35 recreational cyclists report ever suffering from a GOI that was severe enough to interfere with cycling or require treatment or seek medical advice from a health professional. These GOIs affected mostly the knee, lower back and shoulder and $>37 \%$ of injuries were severe enough to affect training and competition. Of concern is that almost 50\% of cyclists with GOIs report symptom duration $>12$ months. This is especially applicable to GOICs of the hip/groin/pelvis, and head/ 
neck/face and lower back. We suggest that further research should focus on determination of the risk factors related with GOIs in recreational cyclists to design and implement effective injury prevention programs. Education of health professionals on the appropriate diagnosis and treatment of GOIs is also recommended.

\section{Findings}

- About 2.8\% (1 in 35) of recreational cyclists report ever suffering from a GOI that is severe enough to interfere with cycling or require treatment or seek medical advice from a health professional.

- GOIs in recreational cyclists affect mostly the knee, lower back and shoulder.

- $>37 \%$ of GOIs in recreational cyclists are severe enough to affect training and competition.

- Almost $50 \%$ of cyclists with GOIs report a symptom duration $>12$ months, perhaps indicating that an appropriate diagnosis was not made, or treatment was ineffective.

\section{Implications}

- To our knowledge, the SAFER XIII study is the largest study documenting the epidemiology and clinical characteristics of GOIs in recreational cyclists.

- The data on prolonged symptom duration emphasises the need for cyclists to report injuries timeously, and clinicians to make an accurate diagnosis and institute appropriate treatment earlier.

- These data also form the basis for studies to determine risk factors related to GOIs in recreational cyclists, and this will assist in planning programs to prevent GOIs.

\section{Caution}

- The study population was significantly older, and that male cyclists were significantly more represented in this group. Our results have to be interpreted with this limitation in mind.

- Specific limitations of our study were that our data are selfreported, with a potential for recall bias.

- Diagnoses of injuries could not be verified by clinical assessment or special investigations.

- As this was a cross-sectional study design, we acknowledge that, from our results, we cannot infer cause-effect.

\section{Contributorship}

François du Toit (FDT): study concept, study planning, data cleaning, data interpretation, manuscript (first draft), manuscript editing.

Martin Schwellnus (MS): responsible for the overall content as guarantor, study concept, study planning, data cleaning, data interpretation, manuscript (first draft), manuscript editing, facilitating funding

Paola Wood (PW): study concept, study planning, data interpretation, manuscript (first draft), manuscript editing,

Sonja Swanevelder (SS): study planning, data cleaning, data interpretation, manuscript editing,

Jannelene Killops (JK): study planning, data collection, data interpretation, manuscript editing.

Esme Jordaan (EJ): study planning, data analysis including statistical analysis, data interpretation, manuscript editing.

\section{Data sharing statement}

No additional data are available.

\section{Funding}

IOC Research Center (South Africa) (partial funding).

South African Medical Research Council (partial funding, statistical analysis).

\section{Declaration of competing interest}

The authors declare that there are no competing interests.

\section{Appendix A. Supplementary data}

Supplementary data to this article can be found online at https://doi.org/10.1016/j.ptsp.2020.08.006.

\section{References}

Bahr, R., Clarsen, B., Derman, W., et al. (2020). International Olympic Committee consensus statement: methods for recording and reporting of epidemiological data on injury and illness in sport 2020 (including STROBE Extension for Sport Injury and Illness Surveillance (STROBE-SIIS)). British Journal of Sports Medicine, 54, 372-389.

Barrios, C., Bernardo, N. D., Vera, P., Laiz, C., \& Hadala, M. (2015). Changes in sports injuries incidence over time in world-class road cyclists. Int J Sports Med, 36(3), 241-248.

Barrios, C., Sala, D., Terrados, N., \& Valenti, J. (1997). Traumatic and overuse injuries in elite professional cyclists. Sports Exercise and Injury, 3(4), 176-179.

Bini, R. R., Hume, P. A., Croft, J., \& Kilding, A. (2014). Optimizing bicycle configuration and cyclists' body position to prevent overuse injury using biomechanical approaches. In Biomechanics of cycling (pp. 71-83). Springer.

Borjesson, M., Serratosa, L., Carre, F., Corrado, D., Drezner, J., Dugmore, D. L., et al. (2011). Consensus document regarding cardiovascular safety at sports arenasposition stand from the european association of cardiovascular prevention and rehabilitation (eacpr), section of sports cardiology. European Heart Journal, 32(17), 2119-2124.

Claes, T., Claes, S., De Roeck, J., \& Claes, T. (2015). Prepatellar friction syndrome: A common cause of knee pain in the elite cyclist. Acta Orthopaedica Belgica, 81(4), 614-619.

Clarsen, B., Bahr, R., Heymans, M. W., Engedahl, M., Midtsundstad, G., Rosenlund, L., et al. (2015). The prevalence and impact of overuse injuries in five Norwegian sports: Application of a new surveillance method. Scandinavian Journal of Medicine \& Science in Sports, 25(3), 323-330.

Clarsen, B., Krosshaug, T., \& Bahr, R. (2010). Overuse injuries in professional road cyclists. The American Journal of Sports Medicine, 38(12), 2494-2501.

Clarsen, B., Myklebust, G., \& Bahr, R. (2013). Development and validation of a new method for the registration of overuse injuries in sports injury epidemiology: The oslo sports trauma research centre (ostrc) overuse injury questionnaire. British Journal of Sports Medicine, 47(8), 495-502.

Clarsen, B., Ronsen, O., Myklebust, G., Florenes, T. W., \& Bahr, R. (2014). The oslo sports trauma research center questionnaire on health problems: A new approach to prospective monitoring of illness and injury in elite athletes. British Journal of Sports Medicine, 48(9), 754-760.

Dahlquist, M., Leisz, M. C., \& Finkelstein, M. (2015). The club-level road cyclist: Injury, pain, and performance. Clinical Journal of Sport Medicine, 25(2), 88-94.

Dannenberg, A. L., Needle, S., Mullady, D., \& Kolodner, K. B. (1996). Predictors of injury among 1638 riders in a recreational long-distance bicycle tour: Cycle across Maryland. The American Journal of Sports Medicine, 24(6), 747-753.

De Bernardo, N., Barrios, C., Vera, P., Laíz, C., \& Hadala, M. (2012). Incidence and risk for traumatic and overuse injuries in top-level road cyclists. Journal of Sports Sciences, 30(10), 1047-1053.

Deakon, R. T. (2012). Chronic musculoskeletal conditions associated with the cycling segment of the triathlon; prevention and treatment with an emphasis on proper bicycle fitting. Sports Medicine and Arthroscopy, 20(4), 200-205.

Decalzi, J. F., Narvy, S. J., \& Vangsness, C. T., Jr. (2013). Overview of cycling injuries: Results of a cycling club survey. Orthopedics, 36(4), 287-289.

Dettori, N. J., \& Norvell, D. C. (2006). Non-traumatic bicycle injuries : A review of the literature. Sports Medicine, 36(1), 7-18.

Farrell, K. C., Reisinger, K. D., \& Tillman, M. D. (2003). Force and repetition in cycling: Possible implications for iliotibial band friction syndrome. The Knee, 10(1), 103-109.

Hainline, B., Derman, W., Vernec, A., Budgett, R., Deie, M., Dvorak, J., et al. (2017). International olympic committee consensus statement on pain management in elite athletes. British Journal of Sports Medicine, 51(17), 1245-1258.

Khan, K. M., Thompson, A. M., Blair, S. N., Sallis, J. F., Powell, K. E., Bull, F. C., et al. 
(2012). Sport and exercise as contributors to the health of nations. Lancet, 380(9836), 59-64.

Killops, J., Schwellnus, M., Janse van Rensburg, D. C., Swanevelder, S., \& Jordaan, E. (2020). Medical encounters, cardiac arrests and deaths during a $109 \mathrm{~km}$ community-based mass-participation cycling event: A 3-year study in 102251 race starters-SAFER IX. British Journal of Sports Medicine, 54(10), 605-611.

Kohl, H. W., Craig, C. L., Lambert, E. V., Inoue, S., Alkandari, J. R., Leetongin, G., et al. (2012). The pandemic of physical inactivity: Global action for public health. The Lancet, 380(9838), 294-305.

Kotler, D. H., Babu, A. N., \& Robidoux, G. (2016). Prevention, evaluation, and rehabilitation of cycling-related injury. Current Sports Medicine Reports, 15(3), 199-206.

Lankhorst, N. E., Bierma-Zeinstra, S. M., \& van Middelkoop, M. (2013). Factors associated with patellofemoral pain syndrome: A systematic review. British Journal of Sports Medicine, 47(4), 193-206.

Powers, C. M. Witvrouw, E., Davis, I. S., \& Crossley, K. M. (2017). Evidence-based framework for a pathomechanical model of patellofemoral pain: 2017 patellofemoral pain consensus statement from the 4th international patellofemoral pain research retreat, manchester, UK: Part 3. British Journal of Sports Medicine, 51(24), 1713-1723.

Rotunno, A., Schwellnus, M. P., Swanevelder, S., Jordaan, E., Janse Van Rensburg, D. C., \& Derman, W. (2018). Novel factors associated with analgesic and anti-inflammatory medication use in distance runners: Pre-race screening among 76654 race entrants-SAFER study VI. Clinical Journal of Sport Medicine, 28(5), 427-434.
Schwabe, K., Schwellnus, M., Swanevelder, S., Jordaan, E., Derman, W., \& Bosch, A. (2018). Leisure athletes at risk of medical complications: Outcomes of preparticipation screening among 15,778 endurance runners - SAFER VII. The Physician and Sportsmedicine, 46(4), 405-413.

Schwellnus, M., \& Derman, W. (2014). The quest to reduce the risk of adverse medical events in exercising individuals: Introducing the SAFER (Strategies to reduce Adverse medical events For the ExerciseR) studies. British Journal of Sports Medicine, 48(11), 869-870.

Taunton, J. E., Ryan, M. B., Clement, D. B., McKenzie, D. C., Lloyd-Smith, D. R., \& Zumbo, B. D. (2003). A prospective study of running injuries: The vancouver sun run "in training" clinics. British Journal of Sports Medicine, 37(3), 239-244.

Townes, D. A., Barsotti, C., \& Cromeans, M. (2005). Injury and illness during a multiday recreational bicycling tour. Wilderness and Environmental Medicine, 16(3), 125-128.

Van der Walt, A., Janse van Rensburg, D. C., Fletcher, L., Grant, C. C., \& Van der Walt, A. J. (2014). Non-traumatic injury profile of amateur cyclists. South African Journal of Sports Medicine, 26(4), 119.

Wanich, T., Hodgkins, C., Columbier, J. A., Muraski, E., \& Kennedy, J. G. (2007). Cycling injuries of the lower extremity. Journal of the American Academy of Orthopaedic Surgeons, 15(12), 748-756.

Weiss, B. D. (1985). Nontraumatic injuries in amateur long distance bicyclists. The American Journal of Sports Medicine, 13(3), 187-192.

Wilber, C. A., Holland, G. J., Madison, R. E., \& Loy, S. F. (1995). An epidemiological analysis of overuse injuries among recreational cyclists. Int J Sports Med, 16(3), 201-206. 